\title{
Challenges and possible solutions in cross- disciplinary and cross-sectorial research teams within the domain of e-mental health
}

Tine Nordgreen, Fazle Rabbi, Jim Torresen, Yngvar Sigmund Skar, Frode Guribye, Yavuz Inal, Eivind Flobakk, Jo Dugstad Wake, Suresh Kumar Mukhiya, Amin Aminifar, Sunniva Myklebost, Astri J. Lundervold, Robin Kenter, Asa Hammar, Emilie Nordby, Smiti Kahlon, Ragnhild J. Tveit Sekse, Kristine Fonnes Griffin, Petter Jakobsen, Minh H. Pham, Ulysse Côté-Allard, Farzan Majeed Noori and Yngve Lamo

\begin{abstract}
Purpose - Mental illness presents a huge individual, societal and economic challenges, currently accounting for $20 \%$ of the worldwide burden of disease. There is a gap between the need for and access to services. Digital technology has been proven effective in e-mental health for preventing and treating mental health problems. However, there is a need for cross-disciplinary efforts to increase the impact of e-mental health services. This paper aims to report key challenges and possible solutions for cross-disciplinary and cross-sectorial research teams within the domain of e-mental health.

Design/methodology/approach - The key challenges and possible solutions will be discussed in light of the literature on effective cross-disciplinary research teams.

Findings - Six topics have been key challenges in our cross-disciplinary and cross-sectorial research team: to develop a shared understanding of the domain; to establish a common understanding of key concepts among the project participants; to involve the end-users in the research and development process; to collaborate across sectors; to ensure privacy and security of health data; and to obtain the right timing of activities according to project dependencies.

Research limitations/implications - This study focuses to increase knowledge and training in crossdisciplinary and cross-sectorial research, as this is often referred to as an important tool when developing sustainable solutions for major societal challenges.

Practical implications - This study needs to include theory and skills training in cross-disciplinary research in research training.

Social implications - Cross-disciplinary teams have the potential to address major societal challenges, including more perspectives and more stakeholders than single disciplinary research teams.

Originality/value - Major societal challenges require complex and sustainable solutions. However, there is a lack of knowledge about how cross-disciplinary and cross-sectorial research teams may work productively to solve these challenges. This paper shares experiences regarding the challenges and possible solutions for productive collaboration in cross-disciplinary and cross-sectorial research teams within the domain of e-mental health services.
\end{abstract}

Keywords Innovation, Psychology, Digital intervention, E-mental health, Cross-disciplinary research teams, Technology enabled care

Paper type Case study

\section{Purpose}

Mental illness presents a huge and growing individual, societal and economic challenge, currently accounting for $20 \%$ of the worldwide burden of disease (Vigo et al., 2016). A major challenge in
(Information about the authors can be found at the end of this article.)
Received 1 March 2021

Revised 25 June 2021

Accepted 6 July 2021

(C) Tine Nordgreen, Fazle Rabbi, Jim Torresen, Yngvar Sigmund Skar, Frode Guribye, Yavuz Inal, Eivind Flobakk, Jo Dugstad Wake, Suresh Kumar Mukhiya, Amin Aminifar, Sunniva

Myklebost, Astri J. Lundervold, Robin Kenter, Asa Hammar, Emilie Nordby, Smiti Kahlon, Ragnhild J. Tveit Sekse, Kristine Fonnes Griffin, Petter Jakobsen, Minh H. Pham, Ulysse Côté-

Allard, Farzan Majeed Noori and Yngve Lamo. Published by Emerald Publishing Limited. This article is published under the Creative Commons Attribution (CC BY 4.0) licence. Anyone may reproduce, distribute, translate and create derivative works of this article (for both commercial and non-commercial purposes), subject to full attribution to the original publication and authors. The full terms of this licence may be seen at http://

creativecommons.org/licences/ by/4.0/legalcode 
mental health is the gap between the need for services and the lack of affordable and timely accessible services. More specifically, only 20-30\% of those with mental health problems receive adequate care. The reasons for this treatment gap are stigma, lack of health personnel and the fact that mental health services have not been prioritized in line with somatic health-care services. Digital technology is suggested as a scalable tool that may increase access to care without expanding health expenditures.

Digital technology has been proven effective in e-mental health for preventing and treating mental health problems (Titov et al., 2018; Kahlon et al., 2019). There is convincing evidence that this approach is effective for a wide range of psychological symptoms and disorders (Karyotaki et al., 2017; Ebert et al., 2018) with higher effects and increased guidance (Palmqvist et al., 2007) and similar effects with head-to-head comparisons and face-to-face treatments (Andersson et al., 2015). E-mental health has several advantages, such as scalability that is independent of time and space, as well as therapy at the patient's own pace (Ebert et al., 2018). However, the current digital treatment programs currently used in health-care services are smartphone apps or websites built as silos without the infrastructure required to share across service levels or geographical regions. Moreover, the current treatment programs are lagging with regard to user experience when compared to all the technologies we are surrounded with in our daily lives (Aravinthan et al., 2020; Rabbi et al., 2020).

To increase the impact of internet-delivered treatments, there is a need to develop effective, userfriendly, adaptive and scalable e-mental health interventions that are in line with the state-of-the-art user experience of digital services, such as banking or travel, and accommodate the requirements in the health services for privacy and security besides being compatible with the existing healthcare services. This challenge cannot be handled by one discipline or sector alone, and there is a need for collaboration between stakeholders, including end-users, and various research disciplines such as psychology, psychiatry, machine learning, data modeling and humancomputer interaction (HCl) (Blandford, 2019). In addition, the information technology (IT) businesses are key partners, as they may have the capability to distribute and maintain secure, effective, user-friendly, adaptive and scalable e-mental health services (Ebert et al., 2018; Karekla et al., 2019). In sum, a cross-disciplinary and cross-sectorial research team in this context can identify, explore and provide innovative solutions to major societal challenges, such as increasing access to mental health services through the use of digital technology (Ding et al., 2020).

For cross-disciplinary and cross-sectorial research teams to be part of the solution, teams need to work productively. To do so, the challenges and possible solutions in cross-disciplinary and crosssectorial research should be identified and acknowledged (Ding et al., 2020). A cross-disciplinary research team is here defined as "one that combines and, in some cases, integrates concepts, methods and theories drawn from two or more disciplines" (Ding et al., 2020, p. 2).

\section{Approach}

In this paper, we share our experiences from four years in a cross-disciplinary and cross-sectorial research team in the domain of e-mental health. The overall aim of the project was to improve public mental health by integrating information and communication technologies and psychological treatments through prototyping, evaluating and implementing innovative solutions. The team has conducted studies and experiments on seven clinical cases (depression, cognitive impairment, anxiety, attention deficit hyperactivity disorder, coping after gynecological cancer, coping with COVID-19 and bipolar disorder), across four work packages (health, machine learning, data modeling and $\mathrm{HCl}$ ), seven industry partners, two patient organizations and one e-mental health clinic. The health researchers were all clinicians and had extensive experience with the relevant target groups. This was vital when assessing the clinical needs of patients and basing digital intervention on state-of-the-art evidence-based treatments. Machine learning researchers have focused on collecting and analyzing data and developing algorithms, all vital for moving beyond the state of affairs of the current internet-delivered 
interventions. Data modeling researchers have worked on developing an architecture that fosters interoperability across traditional silos and facilitates the scalability of the interventions. $\mathrm{HCl}$ researchers focused on user involvement and user testing and worked closely with endusers to understand their needs and develop and evaluate the interventions. The industry partners were interested in expanding their target markets by developing digital platforms and digital interventions for public health-care services. The industry partners were both large international corporations and smaller local businesses; both needed in health-care services. The health-care clinic in the project worked with digital mental health-care services as part of their routine care and represented the needs, complexity and constraints of existing health-care services. Finally, the users represented the target groups at an individual level and systemic level to ensure the relevance of the research and innovation activities in the project. The key topics that will be addressed are: developing a shared understanding of the domain across the project, establishing a common understanding of the key concepts among the different participants, involving the end-users in the research and development process, collaborating across sectors, ensuring privacy and security of health data and obtaining the right timing of activities according to dependencies of activities and tasks in the project.

\section{Findings: six main challenges and possible solutions}

1. To develop a shared understanding of the domain

Challenge: To realize and acknowledge the state of affairs in the domain of regular mental healthcare services is challenging in a time where we are surrounded by user-friendly and persuasive technologies where we receive information and offers based on our previous use of digital tools (Karekla et al., 2019). However, the requirements for privacy and security and the existing approaches to procurement in health-care services are major barriers to such levels of personalization and usability. With this background, it is challenging to explain and understand the current state of user-friendly, data-driven and interoperable digital health services (Mukhiya et al., 2019). Consequently, the motivation and ambitions for using novel technology will vary across the fields of expertise, researchers and stakeholder perspectives in the team.

Possible solution: To accommodate the fact that partners with IT expertise find many IT-related problems in the health-care sector trivial, it has been helpful to distinguish between the innovation and research activities that will lead to "digitalization" and the innovation and research activities that will lead to "experimentation." "Digitalization" is defined as the research leading to innovations that are closer to the current state of affairs in the mental health domain and adopt well-established technological solutions. In digitalization, the primary aim is to pave the way for implementing interventions either through health-care services or industry partners. Digitalization subprojects have fewer risks and, consequently, apply less novel technology within an infrastructure that aligns with the current state of affairs. The state-of-the-art clinical trials are the main research approach in digitalization projects. An example of a digitalization subproject clinical trial is a randomized clinical trial of an internet-delivered intervention to increase women's coping ability after treatment for gynecological cancer or for adults with residual cognitive symptoms after major depressive disorder (Myklebost et al., 2021).

"Experimentation" is defined as activities that include developing and using novel technologies such as unprecedented types of data sources or devices or data analyses based on machine learning algorithms. Consequently, these activities have a higher risk and are a long way from innovation and research besides clinical trials and implementation in regular care. Data-oriented experiments and exploratory design and evaluation of novel technologies are the main research approaches in experimentation projects. An example of an experimentation subproject is the development and evaluation of a smartphone app connected to a wearable device for pervasive sensing of physiological signals or a chatbot (Nordberg et al., 2020; Flobak et al., 2018). 
2. To establish a common understanding of key concepts among the project participants

Challenge: Many cross-disciplinary and cross-sectorial research teams have solved the problems of different understandings of key concepts among different project participants by working in parallel or in sequence rather than in integrated research projects and work processes. To foster active collaboration, it may be helpful to develop a project dictionary, that is, the word "implementation" is understood differently by different partners. Health researchers perceive implementation as the process of bringing an intervention from research into regular care. IT researchers understand implementation as the actual development of a software application. For the funding agency, the term "implementation" is understood to be the process of running the project itself. However, a dictionary was not perceived as helpful, as researchers within the same domain also interpreted key concepts differently. For example, IT researchers soon realized that an "intervention" has different meanings depending on which health researcher or clinician you ask. Health researchers soon realized that a "prototype" from the IT perspective may be drawing on a napkin or a clickable application. Therefore, a dictionary was not helpful as each person, despite being in the same domain, had different interpretations of the same terms.

Possible so/ution: Two strategies have been used to address the need for common univocal use of core concepts. First, we aimed to show, demonstrate and visualize inter alia our hypotheses, methods, scientific theories and prototypes among the participants in the team. Monthly joint collaborative meetings with "show and tell" with all research members in the consortium present, facilitate cross-disciplinary communication. In addition, bi-weekly meetings with work-package leaders foster a frequent review of tasks and deliverables in the project. Second, we aimed to work together on research protocols from the very beginning of each subproject, thereby identifying health, $\mathrm{HCl}$, machine learning and modeling research questions. Our experience is that such activities ensure that research questions and relevant personnel from different domains develop common goals and a common language in each subproject. Furthermore, this process ensures that research questions from different disciplines are included in applications for ethical approval.

\section{To involve the end-users in the research and development process}

Challenge: User involvement is made mandatory for health-related research projects by many funding agencies. More importantly, understanding the needs and preferences of end-users is crucial to ensure engaging, acceptable, relevant and feasible interventions. Despite this, there is little use of models or guidelines on how and when end-users should be involved in the health domain, which is predominantly oriented toward quantitative data. Contrarily, $\mathrm{HCl}$ researchers place a strong emphasis on end-user involvement, with a dominant qualitative approach (Blandford et al., 2018). Moreover, there is no consensus regarding who should be included in the required user involvement. Is it "only" the patients or should we also consider health personnel, relatives, researchers and administrative staff as end-users of the interventions?

The lack of common models for user involvement makes it challenging for research from the health and $\mathrm{HCl}$ disciplines to plan and collaborate to understand user needs (Blandford et al., 2019). This highlights the tension across all scientific disciplines between how we should balance knowledge and evidence at a group level (i.e. from clinical trials) and knowledge and evidence at an individual level (i.e. think-aloud interviews).

Possible solution: We applied the person-based approach (PBA; Yardley et al., 2015), to guide the planning, design and development of our new digital interventions. Early in the project, we found this approach to be a helpful method to integrate the above-mentioned disciplines and perspectives concerning user involvement. PBA follows an iterative mixed-method design based on: (1) a review of quantitative data about the efficacy and feasibility of clinical trials and (2) qualitative data about user needs and preferences. In each iteration, new functionality is added to the intervention, followed by evaluation and user testing. Integrating these perspectives from the planning stage of the novel internet intervention provides a common understanding of balancing the existing knowledge base, user needs and psychosocial context. In our experience, this approach resonates with all research partners in the team. In addition, $\mathrm{HCl}$ researchers have

PAGE 244 |JOURNAL OF ENABLING TECHNOLOGIES $\mid$ VOL. 15 NO. 42021 
sought to supplement the PBA approach with methods and perspectives from participatory design (Flobak et al., 2019; Inal et al., 2020).

\section{To collaborate across sectors}

Challenge: Collaboration between the public and private sectors is one of the foundations of many e-health research projects. In these projects, the relevance of the public sector, including research and health services to the private sector and IT companies, is a key requirement. However, the collaboration between IT businesses in the private sector and IT researchers at public research institutions is challenging, as there are large differences in economic incentives, the process from the development to the marked and the use of project results. There may be an extra barrier for IT researchers and IT businesses to collaborate, as there is a gap between the interesting research questions for IT researchers and what is implementable by IT businesses.

Possible solution: An approach to foster collaboration between research and IT businesses is to obtain a common understanding of what tangible products each research project and partner can produce. One way to grasp this is to differentiate tangible deliverables from the research in the following categories: clinical content, software, hardware, data sets, design and infrastructure. Two years into the project, we established separate meetings for deliverables, where all planned, ongoing and completed activities were tracked and categorized in line with the above.

\section{To ensure privacy and security of health data}

Challenge: It is a challenge for IT and health researchers to gain a joint understanding of data privacy and security. For example, there is a major conflicting trade-off between the emphasis in the General Data Protection Regulation on data minimization, while machine learning depends on large quantities of data that are labeled to train systems for high performance (e.g. Aminifar et al., 2019; Aminifar et al., 2021). Understanding each other's data needs across disciplines, and obtaining the data required for health and IT researchers has been difficult. In addition, where health researchers tend to be acutely aware of the sensitive nature of most collected data, IT researchers are used to large, often open-source, data sets. In contrast, health researchers have handled non-sensitive data as sensitive. Navigating the space between sensitive data and data that can be shared freely among different partners with the right anonymizing measures is also a challenge.

Possible solution: To share data safely and efficiently, we have, via the guidance of the hospitals' Data Protection Officer, developed a routine to follow when sharing data. Along with the legal and practical framework in the form of our consortium agreement and data management agreements, we have implemented a data-sharing agreement for researchers to sign to access data (e.g. Jakobsen et al., 2020). However, this has not been automated because the necessary infrastructure is not yet operational.

\section{To obtain the right timing of activities according to project dependencies}

Challenge: A large project such as ours (budget $<5$ million EURO) has several dependencies across disciplines that are not necessarily mapped out in the writing of the project proposal. Dependencies may be as follows: available data for IT researchers require an infrastructure where sensitive data can be shared at an appropriate level (Mukhia et al., 2020). The data were collected through a clinical trial of a newly developed intervention. The intervention was developed with the involvement of end-users. As a consequence of these dependencies, there has been a timing challenge in coordinating activities and available personnel resources in cross-disciplinary research projects. Another timing issue is that of publications. In what order and in what format should the results be published? This is especially relevant in a cross-disciplinary team where the methodology and reporting culture differ across domains (Blandford et al., 2019). For example, while preparing, conducting and reporting from a clinical trial usually takes between five and seven years, a machine learning paper can be submitted within weeks or months after the data are made available. 
In addition, there has been a challenge with the discontinuation of junior researchers. There is a large need for IT competence in many organizations and companies. Thus, we have seen a higher turnover among IT researchers in the project, leaving temporary research positions in favor of permanent and better-paid jobs, challenging the continuity of the work in the project. Younger candidates make up the large proportion of clinical researchers, several with parental leave during the project. This, in combination, has made it hard to first follow up on planned work in the project. Second, established collaborations end regularly, and new ones take time to establish, especially between researchers across disciplines.

Possible solution: Throughout the project, we chose short- and long-term solutions to solve the dependencies above. First, IT researchers have used relevant data from previous projects to train their algorithms and understand the mental health domain (Garcia-Ceja et al., 2018a, b). Second, we used "silos," meaning specific solutions for specific needs. This has been suboptimal, as this solution does not facilitate collecting and sharing large amounts of data across all cases and partners. Consequently, we realized that some of the timing issues could not be solved within the existing project with the existing milestones, budget and personnel.

Regarding publications, we have established a collaborative publication plan where we agree upon the timing of publications, and Ph.D. candidates' needs across all disciplines have been the main priority. Furthermore, we co-authored and contributed to papers across disciplines.

Regarding the discontinuation of junior staff, the senior staff involved in the project have become more important for contributing to the continuation of project-initiated initiatives and plans. However, these initiatives come in addition to the added effort needed to replace people leaving the project, resulting in increased workload compared to if the junior researchers had been more stable in their positions.

\section{Discussion}

The six key challenges and possible solutions in our cross-disciplinary team in the domain of e-mental health described above include the following key topics: understanding the domain, finding a common language, the involvement of end-users, collaboration across sectors, privacy and security and timing of activities. These topics resonate with existing theories (Lindgreen et al., 2019) and previous research, including a recent literature review of empirical studies examining barriers and enablers of effective cross-disciplinary research teams (Ding et al., 2020). The review categorizes the actions that are addressed in the literature at the level of the individual, team and institution. In the following discussion, we address the key topics introduced in this report according to these three levels.

\section{Individual level}

The actions at the individual level highlighted in the recent literature are mainly related to the willingness to learn from others and their willingness to work collaboratively (Ding et al., 2020). In the INTROMAT project, this may be illustrated with doctoral candidates from different fields of expertise working with the same research protocol, data analysis from different research perspectives resulting in joint publications (Garcia-Ceja et al., 2018a, b), all of which rely on a shared understanding of the domain. One example is the collection and analysis of log data as complementary to self-reported clinical data in clinical trials, assumed to improve the knowledge about engagement, adherence and outcomes of internet-delivered interventions.

Especially relevant at the individual level, the shared understanding and willingness to explore and learn new ideas (Ding et al., 2020; Mallaband et al., 2017) have been highlighted with regard to research designs and, more specifically, randomized controlled trials (RCTs), a gold standard in health research. This design provides high-quality data on the clinical efficacy of treatment packages. However, the efficacy of a single-adaptive treatment element cannot be captured in this 
traditional and static research design. This has led us to conduct a micro randomized trial where machine learning and data modeling are applied, as we can explore the effects of adaptive intervention elements such as prompts. However, to present relevant efficacy data for future dissemination and implementation of the treatment packages in health services, the microrandomized trial was a supplement to the more traditional RCT designs.

However, we have also experienced a lack of shared understanding, and consequently collaboration, in the course of the project. This may be exemplified by too little or too late involvement of IT-related research questions in health research projects or a limited collaboration between IT researchers and business partners. For example, clinical partners provided the specification for apps to be developed by industry partners to conduct clinical studies, largely without relevant IT researchers being involved in defining the relevant data for later IT research and technology development.

\section{Team level}

At the team level, good leadership, exemplified by a clear vision, is important for successful crossdisciplinary teams (Ding et al., 2020). The experience of the INTROMAT project is that a clear vision motivates team members to endure frustrations and challenges when it comes to shared understanding and willingness to collaborate. We also want to highlight the importance of a "meaningful" vision, that is, a vision related to a set of shared values. This may be easier to foster in some projects, for example, when working on major societal challenges that directly or indirectly affect most people, such as mental health or global climate change.

The capability to facilitate good communication across disciplines and sectors is also important for cross-disciplinary teams (Ding et al., 2020). In line with the literature (Schönenberg et al., 2017), we find that frequent and regular physical meetings facilitate productive communication and, consequently, collaboration across disciplines. More specifically, discussions regarding user involvement require open discussions about ethics, values and traditions within different disciplines and sectors. Included in this dialog was also training in new methods provided by external parties. We also found that distance in time or geography was associated with less positive outcomes. Moreover, four years into the research project, some key concepts are still not fully understood across all disciplines and sectors.

\section{Institutional level}

At an institutional level, it is vital that both the hosting and partner institutions allocate resources to coordinating and supporting the cross-disciplinary research team in their efforts to solve complex problems (Ding et al., 2020). The hosting institution provided meetings and office facilities for all partners. For example, this has been used weekly by one of our industry partners who have sat together with health researchers. On the partners' side, most research partners were willing to participate in quarterly steering group meetings, including heads of departments of all workpackage leaders and key industry partners. This is vital when prioritizing tasks, deliverables and milestones in the project, including the timing of dependencies.

Both the funder and host institutions have provided vital support throughout the project, both critical for cross-disciplinary teams (Ding et al., 2020). In addition to the five-year funding, the funder has been accessible for consultations in difficult matters and has been flexible concerning annual revisions of the deliverables and corresponding budgets. The funders have also given project access to national and international platforms where the research can be disseminated. The hosting institution, a university hospital, has provided strong weekly support. In our experience, university hospitals are not only host institutions but also one of the major stakeholders in terms of the major societal challenge of mental health. The latter promotes clear expectations for deliverables from cross-disciplinary teams. 
One key partner left the consortium early in the project, mainly because the expectations for deliverables (publications) by one of the partner institutions exceeded what the cross-collaborative research activities could produce. The fact that single-discipline research progresses faster than cross-disciplinary research teams is recognized in the literature (Trussel et al., 2017) and should therefore be addressed when building cross-disciplinary research teams (Mallaband et al., 2017).

Finally, we want to emphasize that the interplay between the individual, team and institutional levels has been a major contributor to handling the challenges and identifying possible solutions in the INTROMAT cross-disciplinary research project. For example, the support and enthusiasm of the steering group members with institutional representation has contributed to handling frustrations and disagreements at the individual and team levels. In addition, the support from the funder and a shared vision among all team members for increasing access to mental health services through digital technology has allowed for open discussions about the challenges and the search for solutions in our cross-disciplinary research team.

\section{Implications}

Cross-disciplinary innovation and research are important tools for solving major societal challenges. However, there are many barriers for cross-disciplinary and sectorial teams to work effectively (Ding et al., 2020; Pellegrino and Musy, 2017). Based on our experiences, previous research (Ding et al., 2020), and funding agencies' incentives, there is a need to develop research training programs relevant to this approach. In addition, there is a need for hosting institutions to not only support the application of large grants that involve cross-disciplinary teams but also to support such teams during the project period (Pellegrino and Musy, 2017). Taken together, this has the potential to enhance the impact of research and innovation when solving major societal challenges.

\section{Acknowledgments}

The authors want to thank all participants included in our clinical trials. The authors also want to thank all students who has participated in the INTROMAT- project.

Funding: This paper is from the project Introducing Mental health through Adaptive Technology (http://www.intromat.no) funded by the Norwegian Research Council (NFR: 259293).

\section{References}

Aminifar, A., Lamo, Y., Pun, K. and Rabbi, F. (2019), "A practical methodology for anonymization of structured health data", Proceedings of the 17th Scandinavian Conference on Health Informatics.

Aminifar, A., Rabbi, F., Pun, K. and Lamo, Y. (2021), "Privacy preserving distributed extremely randomized trees", Proceedings of the 36th Annual ACM Symposium on Applied Computing.

Andersson, G., Topooco, N., Havik, O. and Norgreen, T. (2015), "Internet-supported versus face-to-face cognitive behavior therapy for depression", Expert Review of Neurotherapeutics, Vol. 16, doi: 10.1586/ 14737175.2015.1125783.

Aravinthan, Y., Kenter, R., Lamo, Y., Kaldo, V. and Nordgreen, T. (2020), "Internet-delivered mental health treatment systems in Scandinavia-A usability evaluation", Internet Interventions, Vol. 7, p. 3.

Blandford, A. (2019), "HCl for health and wellbeing: challenges and opportunities", International Journal of Human-Computer Studies, Vol. 131, pp. 41-51, doi: 10.1016/j.ijhcs.2019.06.007, available at: https://www. sciencedirect.com/science/article/pii/S1071581919300771.

Blandford, A., Gibbs, J., Newhouse, N., Perski, O., Singh, A. and Murray, E. (2018), "Seven lessons for interdisciplinary research on interactive digital health interventions", Digital Health, Vol. 4, doi: 10.1177/ 2055207618770325

Ding, Y., Pulford, J. and Bates, I. (2020), "Practical actions for fostering cross-disciplinary global health research: lessons from a narrative literature review", BMJ Global Health, Vol. 5, e002293.

PAGE 248 JOURNAL OF ENABLING TECHNOLOGIES $\mid$ VOL. 15 NO. 42021 
Ebert, D.D., Daele, T., NordgreenKarekla, T.M., Compare, T.A., Zarbo, C., Brugnera, A., Oeverland, S., Trebbi, G., JensenKaehlke, K.L.F. and on behalf of the EFPA E-Health Taskforce, (2018). "Internet- and mobile-based psychological interventions: applications, efficacy, and potential for improving mental health a report of the EFPA E-health taskforce", European Psychologist, Vol. 23 No. 2, pp. 167-187, doi: 10.1027/ 1016-9040/a000318.

Flobak, E., Jensen, D.A., Lundervold, A.J., Nordgreen, T., Chen, L.-H. and Guribye, F. (2018), "Towards technology-based interventions for improving emotional and cognitive control", Extended Abstracts of the 2018 CHI Conference on Human Factors in Computing Systems (CHI EA '18), Association for Computing Machinery, New York, NY, pp. 1-6, Paper LBW049, doi: 10.1145/3170427.3188603.

Flobak, E., Wake, J.D., Vindenes, J., Kahlon, S., Nordgreen, T. and Guribye, F. (2019), "Participatory design of VR scenarios for exposure therapy", Proceedings of the 2019 CHI Conference on Human Factors in Computing Systems (CHI '19). ACM, New York, NY, Paper 569, p. 12, doi: 10.1145/3290605.3300799.

Garcia-Ceja, G., Alejandro, E., Riegler, M., Jakobsen, P., Tørresen, J., Nordgreen, T., Ødegaard, K.J. and Fasmer, O.B. (2018a), "Motor activity based classification of depression in unipolar and bipolar patients", IEEE International Symposium on Computer-Based Medical Systems, Karlstad, pp. 316-321.

Garcia-Ceja, E., Riegler, M., Jakobsen, P., Tørresen, J., Nordgreen, T., Oedegaard, K.J. and Fasmer, O.B. (2018b), "Depression: a motor activity database of depression episodes in unipolar and bipolar patients", Proceedings of the 9th ACM Multimedia Systems Conference, ACM, Amsterdam, pp. 472-477, doi: 10. $1145 / 3204949.3208125$.

Inal, A., Wake, J.D., Guribye, F. and Nordgreen, T. (2020), "Usability evaluations of mobile mental health technologies: systematic review", Journal of Medical Internet Research, Vol. 22, p. 1.

Jakobsen, P., Garcia-Ceja, E., Riegler, M., Stabell, L.A., Nordgreen, T., Torresen, J., Fasmer, O.B. and Oedegaard, K.J. (2020), "Applying machine learning in motor activity time series of depressed bipolar and unipolar patients compared to healthy controls. Applying machine learning in motor activity time series of depressed bipolar and unipolar patients compared to healthy controls", PLoS One, Vol. 15 No. 8, e0231995, doi: 10.1371/journal.pone.0231995.

Kahlon, S., Lindner, P. and Nordgreen, T. (2019), "Virtual reality exposure therapy for adolescents with fear of public speaking: a non-randomized feasibility and pilot study", Child and Adolescent Psychiatry and Mental Health, Vol. 13 No. 1, pp. 1-10.

Karekla, M., Kasinopoulos, O., Neto, D.D., Ebert, D.D., Daele, T.M., Nordgreen, T., Höfer, S., Oeverland, S. and Jensen, K.L. (2019), "Best practices and recommendations for digital interventions to improve engagement and adherence in chronic illness sufferers", European Psychologist, Vol. 24 No. 1, pp. 49-67.

Karyotaki, E., Riper, H., Twisk, J., Hoogendoorn, A., Kleiboer, A., Mira, A., Mackinnon, A., Meyer, B., Botella, C., Littlewood, E., Andersson, G., Christensen, H., Klein, J.P., Schröder, J., Bretón-López, J., Scheider, J., Griffiths, K., Farrer, L., Huibers, M.J., Phillips, R., Gillbody, S., Moritz, S., Berger, T., Pop, V., Spek, V. and Cuijpers, P. (2017), "Efficacy of self-guided internet-based cognitive behavioral therapy in the treatment of depressive symptoms: a meta-analysis of individual participant data", JAMA Psychiatry, Vol. 74 No. 4, pp. 351-359, doi: 10.1001/jamapsychiatry.2017.0044.

Lindgreen, A., Di Benedetto, A., Brodie, R. and Naudé, P. (2019), "How to build great research groups", Industrial Marketing Management, Vol. 81, doi: 10.1016/j.indmarman.2019.07.009.

Mallaband, B., Wernham, G., Buchanan, K., Staddon, S., Mogles, N. and Gabe-Thomas, E. (2017), "The reality of cross-disciplinary energy research in the United Kingdom: a social science perspective", Energy Research and Social Science, Vol. 25, pp. 9-18, doi: 10.1016/j.erss.2016.11.001.

Mukhiya, S.K., Rabbi, F., Pun, K., Rutle, A. and Lamo, Y. (2019), "A GraphQL approach to healthcare information exchange with HL7 FHIR", Procedia Computer Science, Vol. 160, pp. 338-345, ISSN 1877 0509, doi: 10.1016/j.procs.2019.11.082, available at: https://www.sciencedirect.com/science/article/pii/ S187705091931782X.

Mukhiya, S.K., Wake, J., Inal, Y., Pun, K. and Lamo, Y. (2020), "Adaptive elements in internet-delivered psychological treatment systems: systematic review", Journal of Medical Internet Research, Vol. 22 No. 11, e21066, doi: 10.2196/21066.

Myklebost, S.B., Nordgreen, T. and Hammar, A. (2021), "An open pilot study of an internet-delivered intervention targeting self-perceived residual cognitive symptoms after major depressive disorder", Applied Neuropsychology: Adult. doi: 10.1080/23279095.2021.1901706.

Nordberg, O.E., Wake, J.D., Nordby, E.S., Flobak, E., Nordgreen, T., Mukhiya, S.K. and Guribye, F. (2020), "Designing chatbots for guiding online peer support conversations for adults with ADHD", in Følstad, A., et al.

VOL. 15 NO. 42021 JOURNAL OF ENABLING TECHNOLOGIES $\mid$ PAGE 249 
(Ed.), Chatbot Research and Design. Conversations 2019: Lecture Notes in Computer Science, Springer, Vol. 11970, doi: 10.1007/978-3-030-39540-7_8.

Palmqvist, B., Carlbring, P. and Andersson, G. (2007), "Internet-delivered treatments with or without therapist input: does the therapist factor have implications for efficacy and cost?", Expert Review of Pharmacoeconomics and Outcomes Research, Vol. 7 No. 3, pp. 291-297, doi: 10.1586/14737167.7.3.291.

Pellegrino, M. and Musy, M. (2017), "Seven questions around interdisciplinarity in energy research", Energy Research and Social Science, Vol. 32, pp. 1-12, doi: 10.1016/j.erss.2017.07.007, available at: https://www. sciencedirect.com/science/article/pii/S221462961730230X.

Rabbi, F., Wake, J.D. and Nordgreen, T. (2020), "Reusable data visualization patterns for clinical practice", in Babur, Ö., Denil, J. and Vogel-Heuser, B. (Eds), Systems Modelling and Management ICSMM 2020. Communications in Computer and Information Science, Springer, Cham, Vol. 1262, doi: 10.1007/978-3030-58167-1_5.

Schönenberg, R., Boy, J., Hartberger, K., Schumann, C., Guggenberger, G., Siebold, M., Lakes, T., Lamparter, G., Schindewolf, M., Schaldach, R., Nendel, C., Hohnwald, S., Meurer, K., Gerold, G. and Klingler, M. (2017), "Experiences of inter- and transdisciplinary research - a trajectory of knowledge integration within a large research consortium", Erdkunde, Vol. 71, pp. 177-193, doi: 10.3112/erdkunde. 2017.03.02.

Titov, N., Blake, D., Nielssen, O., Staples, L., Hadjistavropoulos, H., Nugent, M., Adlam, K., Nordgreen, T., Bruvik, K.H., Hovland, A., Repål, A., Mathiasen, K., Kraepelien, M., Blom, K., Svanborg, C., Lindefors, N. and Kaldo, V. (2018). "ICBT in routine care: a descriptive analysis of successful clinics in five countries", Internet Interventions, Vol. 13, pp. 108-115, doi; 10.1016/j.invent.2018.07.006.

Trussell, D.E., Paterson, S., Hebblethwaite, S., Xing, T.M.K. and Evans, M. (2017), "Negotiating the complexities and risks of interdisciplinary qualitative research", International Journal of Qualitative Methods, Vol. 16 No. 1, doi: 10.1177/1609406917711351.

Vigo, D., Thornicroft, G. and Atun, R. (2016), "Estimating the true global burden of mental illness", The Lancet Psychiatry, Vol. 3 No. 2, pp. 171-178.

Yardley, L., Morrison, L., Bradbury, K. and Muller, I. (2015), "The person-based approach to intervention development: application to digital health-related behavior change interventions", Journal of Medical Internet Research, Vol. 17 No. 1, e30, doi: 10.2196/jmir.4055.

\section{Author affiliations}

Tine Nordgreen is based at the Division of Psychiatry, Haukeland University Hospital, Bergen, Norway and Faculty of Medicine and Dentistry, University of Bergen, Bergen, Norway.

Fazle Rabbi is based at the Department of Computer Science, Electrical Engineering, and Mathematical Sciences, Western Norway University of Applied Sciences, Bergen, Norway.

Jim Torresen is based at the Department of Informatics, Faculty of Mathematics and Natural Sciences, University of Oslo, Oslo, Norway.

Yngvar Sigmund Skar is based at the Helse Vest IKT AS, Bergen, Norway.

Frode Guribye, Yavuz Inal and Eivind Flobakk are all based at the Department of Information Science and Media Studies, Faculty of Social Science, University of Bergen, Bergen, Noway.

Jo Dugstad Wake is based at the NORCE Norwegian Research Centre AS, Bergen, Norway.

Suresh Kumar Mukhiya and Amin Aminifar are both based at the Department of Computer Science, Electrical Engineering, and Mathematical Sciences, Western Norway University of Applied Sciences, Bergen, Norway.

Sunniva Myklebost and Astri J. Lundervold are both based at the Department of Biological and Medical Psychology, Faculty of Psychology, University of Bergen, Bergen, Norway.

Robin Kenter is based at the Department of Clinical Psychology, Faculty of Psychology, University of Bergen, Bergen, Norway.

Assa Hammar is based at the Department of Biological and Medical Psychology, Faculty of Psychology, University of Bergen, Bergen, Norway.

PAGE 250 JOURNAL OF ENABLING TECHNOLOGIES $\mid$ VOL. 15 NO. 42021 
Emilie Nordby and Smiti Kahlon are both based at the Division of Psychiatry, Haukeland University Hospital, Bergen, Norway.

Ragnhild J. Tveit Sekse is based at the Department of Clinical Psychology, Faculty of Psychology, University of Bergen, Bergen, Norway.

Kristine Fonnes Griffin is based at the Haukeland Universitetssjukehus, Bergen, Norway.

Petter Jakobsen is based at the Norment, Division of Psychiatry, Haukeland University Hospital, Bergen, Norway and Department of Clinical Medicine, University of Bergen, Bergen, Norway.

Minh H. Pham, Ulysse Côté-Allard and Farzan Majeed Noori are all based at the Department of Informatics, Faculty of Mathematics and Natural Sciences, University of Oslo, Oslo, Norway.

Yngve Lamo is based at the Department of Computer Science, Electrical Engineering, and Mathematical Sciences, Western Norway University of Applied Sciences, Bergen, Norway.

\section{Corresponding author}

Tine Nordgreen can be contacted at: tine.nordgreen@uib.no

For instructions on how to order reprints of this article, please visit our website: www.emeraldgrouppublishing.com/licensing/reprints.htm Or contact us for further details: permissions@emeraldinsight.com 